\title{
Patterns and Transitions to Instability in an Intraspecific Competition Model with Nonlocal Diffusion and Interaction
}

\author{
O. Aydogmus* \\ Department of Economics, Social Sciences University of Ankara, 06100 Ulus-Ankara, Turkey
}

\begin{abstract}
We consider an intraspecific resource competition model with asymmetric nonlocal dispersal and interaction. Both interaction and dispersal are modeled using convolution integrals. We introduce two parameters for each convolution kernel describing the range of nonlocality and the extent of asymmetry. It is shown that the spatially homogenous equilibrium of this model becomes unstable for sufficiently small diffusion rates by performing a linear stability analysis. Our numerical observations indicate that traveling and stationary wave type patterns arise near the stability boundary. Away from the stability boundary, solutions to the model exhibit colony formation i.e. one can observe formation of a new colony island just ahead of a vanishing stationary wave island. We further analyze the behavior of solutions to the model near the stability boundary using the techniques of weakly nonlinear analysis. We obtain a Stuart-Landau type equation and give its parameters in terms of Fourier transforms of the kernels. This analysis allows us to study the change in amplitudes of the solutions with respect to ranges of nonlocalities and extents of asymmetries of two kernel functions. We show that both continuous and discontinuous transitions from disordered behavior to ordered one are possible. We also verify these results numerically.
\end{abstract}

Keywords and phrases: nonlocal diffusion, nonlocal competition, pattern formation, weakly nonlinear analysis.

Mathematics Subject Classification: 92B05, 37N25, 34K09, 37G99

\section{Introduction}

The model derived and investigated here is a one dimensional intraspecific competition model which considers nonlocally interacting and dispersing individuals competing for sparse resources. This model can be expressed as a single integro-differential equation (IDE) with two kernel functions. Hence, we consider the frequency of the species, $u(x, t)$, at spatial location $x$ and time $t$ satisfying the following IDE:

$$
u_{t}=\beta\left(K_{1} * u-u\right)+u\left(1-K_{2} * u\right)
$$

\footnotetext{
*Corresponding author. E-mail: ozgur.aydogmus@asbu.edu.tr
} 
where $\beta$ is the nonlocal diffusion rate and the convolution terms are given by $K_{i} * u(x, t)=\int_{\mathbb{R}} K_{i}(x-$ $y) u(y, t) d y$ for $i=1,2$. In this study, we consider the kernel functions satisfying the following set of assumptions:

$$
K_{i} \geq 0 \text { and } \int_{\mathbb{R}} K_{i}(x) d x=1
$$

for $i=1,2$. Biologically, the meaning of the nonlocal competition term $\left(K_{2} * u\right)$ is that consumption of resources at a spatial location for mobile individuals does not only depend on the population density at that point but also on the weighted average of the population at the same point. Here we would like to note that the destabilization of the space homogenous solution to (1.1) depends heavily on the interaction kernel, $K_{2}$. Thus, we impose the following condition on the interaction kernel:

C: The real part of the Fourier transform of the interaction kernel takes negative values.

On the other hand the dispersal kernel, $K_{1}$, specifies the mobility of individuals (see for example $[11,12]$ ). Here, our aim is to investigate how asymmetric dispersal and interaction affect the formation of patterns and transitions to instabilities.

There are several studies considerig the same model with local dispersal which is given as follows:

$$
v_{t}=\tilde{\beta} \Delta v+v(1-K * v)
$$

where $\tilde{\beta}$ is the local diffusion rate and the convolution term is given as above. The model is a generalization of well-known Fisher equation which is called as non-local Fisher equation or competitive Lotka-Volterra model in the literature [14]. Equation (1.3) models individuals from the same species with nonlocal interaction and local dispersal. It is studied in terms of nonmonotone traveling waves [8]. Equation (1.3) admits a single spatially homogenous equilibrium. It has been reported that this equilibrium may become unstable for small diffusion rates $[4,5]$ if the nonlocal interaction term is taken as a symmetric step function. The effect of asymmetric interaction kernels is also studied in $[6,7,14]$ and it is concluded that the destabilization of the space homogenous equilibrium is possible for small diffusion parameters under the condition $\mathbf{C}$.

A two dimensional nonlocal interspecific competition model with a random diffusion term is considered in [1]. The model was explored for symmetric kernel functions by performing a linear stability analysis in [15]. The interspecific competition with asymmetric nonlocal interactions was studied in [17]. They find solutions exhibiting colony formation where nearby colony islands are formed as parent islands vanish. In addition, their numerical simulations indicate that the transition to instability is supercritical, i.e. small-amplitude sinusoidal oscillations near the stability boundary are observed. This implies continuous transitions from disordered to ordered behavior.

In population dynamics and ecology, reaction-diffusion equations (RDEs) are one of the most widely employed tools in modeling locally diffusing individuals $[2,9]$. However, by using these equations, one assumes that the individuals' motion is governed by a simple random walk. Since this assumption is too restrictive, non-local reaction diffusion equations have come in the picture recently (see for example, [11]). These equations are useful to model more general movement patterns than the local diffusion and advection [12]. In section 2, we derive (1.1) as a nonlocal analog of intraspecific competition model studied in $[4-8,14]$ by considering nonlocally diffusing individuals.

Here we would like to note that performing a linear stability analysis is useful to find conditions for formation of patterns, which informs us about the value of the unstable wavenumber and the parameters for which destabilization of the space homogenous solution occurs. To further investigate the complex structure of the attractors, one can employ the classical Stuart-Landau (SL) theory [16]. The theory is widely applied to RDE models in biology [13] and physics [20]. Recently it has been applied to nonlocal aggregation models $[3,19]$ and it was reported that the transition to instability is subcritical i.e. near the stability boundary, one can observe large oscillations depending on the initial condition. In other words, transition from disordered to ordered behavior is discontinuous. 
The aim of the paper is twofold. Firstly, we focus on the linear stability analysis of equation (1.1). We show that instabilities occur under condition $\mathbf{C}$ for small diffusion rates $\beta$. Our numerical computations indicate that one can observe traveling and stationary wave type behavior near the stability boundary. In addition, we give two numerical examples for which solutions exhibit continuous and discontinious transitions to instabilities near stability boundaries. Beyond the stability boundary, our computations show that solutions to (1.1) exhibit colony formation. In other words, one can observe the formation of new colony islands as stationary wave type parent islands vanish. Secondly, we employ weakly nonlinear analysis of the equation to study the transitions to instabilities. This analysis allows us to identify the parameter regions where transitions to instabilities are supercritical and subcritical. In addition, the analysis gives information about the change in amplitudes of solutions to the equation. We verify our findings numerically using the above mentioned examples.

The paper is organized as follows. In the next section we derive the nonlocal reaction-diffusion equation with nonlocal interaction and dispersal. In section 3 we show results of linear stability analysis and numerically investigate the patterns whenever the space homogenous solution is unstable. In section 4 we obtain and analyze a Stuart-Landau type equation for the amplitudes of solutions to equation (1.1). We conclude and summarize our findings in Section 5

\section{Model Derivation}

We consider a population of one species in a one-dimensional habitat and derive equation (1.1). Note that the method used here can be generalized to $n$ dimensions easily. We denote the density of the species by a single function of the spatial location $x$ and time $t$. Divide our habitat $\mathbb{R}$ into contiguous sites of length $\delta x$ and discretize time into steps of size $\delta t$. Let $\tilde{u}(i, t)$ be the density of the population at site $i$ and time $t=p \cdot \delta t$ for $p \in \mathbb{N}$. Assume that the rate at which organisms leaves the site $i$ and goes to site $j$ is constant. Denote this constant by $k_{1}(i, j)$ measuring the transit rate from $i$ to $j$ during the time interval $[t, t+\delta t]$. Then we obtain the number of individuals leaving site $i$ during this time interval as follows:

$$
S_{1}=\sum_{\substack{j=-\infty \\ j \neq i}}^{\infty} k_{1}(i, j) \tilde{u}(i, t)(\delta x)^{2} \delta t .
$$

Conversely the number of individuals coming to site $i$ is given by

$$
S_{2}=\sum_{\substack{j=-\infty \\ j \neq i}}^{\infty} k_{1}(j, i) \tilde{u}(j, t)(\delta x)^{2} \delta t .
$$

Suppose that our reaction term is of the following form:

$$
F(\tilde{u}(i, t))=(B-D) \tilde{u}(i, t) \delta x \delta t
$$

denoting the per capita net reproduction rate at site $i$. Then we obtain the change of density at site $i$ between times $t$ and $t+\delta t$ as follows:

$$
\tilde{u}(i, t+\delta t)-\tilde{u}(i, t)=S_{2}-S_{1}+F(\tilde{u}(i, t)) .
$$

By taking the limits as $\delta t \rightarrow 0$ and $\delta x \rightarrow 0$ we get the following equation:

$$
\bar{u}_{t}(x, t)=\int_{\mathbb{R}}\left[K_{1}(y, x) \bar{u}(y, t)-K_{1}(x, y) \bar{u}(x, t)\right] d y+\bar{u}(x, t)(B-D) .
$$

We define the reaction term following [7]. To be able to obtain logistic reaction term, we assume that the birth rate $B$ is constant and the death rate is given by

$$
D=b+q \int_{\mathbb{R}} K_{2}(x, y) \bar{u}(y, t) d x .
$$


where $b$ describes the natural death and the second term increases the death rate proportional to the average number of individuals around location $y$. For the details of the derivation of this death function, see [7]. Suppose the kernel functions satisfy $K_{i}(x, y)=K_{i}(x-y)$. and $\int_{\mathbb{R}} K_{i}(x) d x=1$ for $i=1,2$. The former assumption implies that transitions between two locations $x$ and $y$ depend only on the distance between them. The later guarantees that the density of the population at location $y$ before the transition is equal to the sum of all transitions from $y$. By equation (2.1), one can easily get

$$
\bar{u}_{\bar{t}}=K_{1} * \bar{u}-\bar{u}+\bar{u}\left(\tilde{B}-p K_{2} * \bar{u}\right)
$$

where $\tilde{B}=B-b$.

Rescaling $\bar{u}=\tilde{B} u / p$ and $\bar{t}=t / \tilde{B}$ in $(2.2)$ gives (1.1) with $\beta=1 / \tilde{B}$.

\section{Linear Stabilty Analysis and Computed Patterns}

We perform a linear stability analysis to examine the effect of the diffusion rate $\beta$ and the kernels $K_{1}$ and $K_{2}$ on destabilization of the space homogenous equilibrium of (1.1). In addition, we numerically investigate the spatiotemporal patterns arising from (1.1) such as traveling/stationary waves and colony formation.

\subsection{Linear Stability Analysis}

Without spatial effects i.e. $K_{1}=K_{2}=\delta$ where $\delta$ is the Dirac delta functional, (1.1) reduces to logistic equation with stable solution $u=1$.

To investigate necessary conditions for pattern formation, we linearize the equation by taking $u=$ $1+\varepsilon \check{u} e^{\lambda t}$, where $\check{u}(x)$ is a spatial perturbation term. Substituting this ansatz to the equation (1.1) gives the following first order relation

$$
\lambda \check{u}=\beta\left(K_{1} * \check{u}-\check{u}\right)-K_{2} * \check{u} .
$$

By taking the Fourier transform of both sides, one can obtain

$$
\lambda(\beta, k)=\beta\left(\hat{K}_{1}(k)-1\right)-\hat{K}_{2}(k)
$$

where $\hat{K}_{i}(k)$ is the Fourier transform of the kernel $K_{i}$ for $i=1,2$. Here we would like to note that the Fourier transforms of these kernels can take complex values since the kernel functions are not necessarily symmetric. Therefore, we introduce notations $\mathcal{R}[z]$ and $\mathcal{I}[z]$ to denote real and imaginary parts of a complex number $z$, respectively.

Note also that

$$
\hat{K}_{i}(0)=\int_{\mathbb{R}} K_{i}(y) d y=1
$$

for $i=1,2$. Thus, $\mathcal{R}\left(\hat{K}_{1}(k)-1\right) \leq 0$ for the dispersal kernel. This implies that we need to require $\mathcal{R}\left[\hat{K}_{2}(k)\right] \leq 0$ for some $k \in \mathbb{R}$ to destabilize steady state $u=1$.

This analysis can also be conducted for periodic habitats. With periodic boundary conditions in a box $I=[0, L]$, similar results can be obtained by taking spatially periodic perturbations i.e. $\breve{u}(x)=e^{i k_{n} x}$ where $k_{n}=\frac{2 n \pi}{L}$ denotes the finite wavelengths for $n \in \mathbb{N}$. In this case, it is required that $\mathcal{R}\left[\hat{K}_{2}\left(k_{n}\right)\right] \leq 0$ for some $n \in \mathbb{N}$. Suppose that $\beta_{0}$ is the critical value of the nonlocal diffusion coefficient such that spatially homogenous equilibrium 1 becomes unstable for any $\beta<\beta_{0}$, i.e. $\mathcal{R}\left[\lambda\left(\beta, k_{n}\right)\right]>0$ for some $n \in \mathbb{N}$. The critical wavenumber $k_{c}=k_{n}$ is given by $n \in \mathbb{N}$ satisfying $\mathcal{R}\left[\lambda\left(\beta_{0}, k_{n}\right)\right] \geq 0$ and is called the most unstable wavenumber.

In [7], it has been shown that solutions to nonlocal Fisher equation exhibit pattern formation under the condition C. Hence, symmetric uniform kernels have been used widely (see for example $[4,5,7]$ ). Following recent works $[15,17]$, we assume that each convolution term depends on two parameters: $d_{i}>0$ and $a_{i}$ for $i=1,2$. Here $d_{i}$ characterizes the range of nonlocality and $a_{i}$ is the extent of asymmetry for the kernel 
function $K_{i}$. Whenever $a_{i}=0$, the corresponding kernel becomes symmetric. As an example, one can consider the family of step functions as follows:

$$
\mathcal{K}_{(d, a)}(x)= \begin{cases}\frac{1}{2 d} & \text { if }|x-a| \leq d \\ 0 & \text { if }|x-a|>d\end{cases}
$$

We note that the condition $\mathbf{C}$ holds for the kernel family $\mathcal{K}_{(d, a)}$ even for $a=0$. Thus, instabilities occur for diffusion rates satisfying $\beta \leq \beta_{0}$. On the other hand, one can also use Gaussian or Laplacian families [17]. To destabilize the space homogenous equilibrium, the interaction kernel should be asymmetric ( $\left.a_{2} \neq 0\right)$ whenever Gaussian or Laplacian families are used. In our numerical studies, we concentrate on the family of uniform kernel functions (3.3).

\subsection{Computed Patterns}

We start by computing the solutions to (1.1) around the nonlocal stability boundary $\beta_{0}$ and observe patterns like traveling and stationary waves verifying results obtained by linear stability analysis in Section 3.1. We further investigate the solutions to the equation away from the stability boundary and observe colony formation.

In our numerical simulations, we consider (1.1) with wrap around boundary conditions. Both convolution terms $K_{1} * u$ and $K_{2} * u$ are approximated by fast Fourier transform. Equation (1.1) is discretized by a forward time finite difference scheme with time interval $\Delta t=0.005$. We used the discretization of the space variable $0 \leq x \leq L$ with a mesh interval of $\Delta x=0.001$ and $L=10$.

The critical value of the parameter, $\beta_{0}$, can be obtained by solving the following root finding problem:

$$
\max _{\mathbf{k}} \mathcal{R}[\lambda(\beta, \mathbf{k})]=0
$$

where $\mathbf{k}=\left(k_{1}, k_{2}, \ldots\right)$. For concreteness, we focus on the following set of parameters:

P1: $K_{1}=K_{2}=\mathcal{K}_{(1,0)}$,

P2: $K_{1}=\mathcal{K}_{(1,0)}$ and $K_{2}=\mathcal{K}_{(1.88,-0.71)}$,

P3: $K_{1}=\mathcal{K}_{(1,0.1)}$ and $K_{2}=\mathcal{K}_{(1,-0.3)}$,

with the initial condition specified by

$-i\left(\alpha_{0}\right):=u_{0}(x)=1+\alpha_{0} \cos \left(k_{c} x\right)$

where $2 \alpha_{0}$ is the amplitude of the initial condition. We use the parameters given by $\mathbf{P} \mathbf{1}$ and $\mathbf{P} \mathbf{2}$ to study traveling and stationary waves and $\mathbf{P} \mathbf{3}$ is used to observe colony formation beyond the stability boundary.

\subsubsection{Stationary and traveling waves}

Here we focus on instabilities and formation of patterns. Our numerical simulations indicate that only traveling and stationary waves can be observed near stability boundaries. Note also that similar patterns were observed for nonlocal Fisher equation in [7].

Near the stability boundaries, we consider two examples as follows: (1) stationary waves, in Figure 1 (a), were obtained for the parameters $\mathbf{P} 1$, initial condition $i(0.01)$ and $\beta=0.1768$. For above specified kernel functions, the critical value of the nonlocal diffusion rate is $\beta_{0}=0.1778$. (2) Figure 1 (b) shows periodic traveling waves. These patterns were obtained for the kernel functions with parameters $\mathbf{P 2}$, initial condition $i(0.01)$ and the diffusion rate is taken as the critical nonlocal diffusion rate i.e. $\beta=\beta_{0}=0.0910$.

The third example given in Figure 1 (c) illustrates stationary waves with a smaller diffusion rate $\beta=0.0910$ and same initial condition $i(0.01)$. As seen from the colorbar presented in panel (c), this smaller diffusion rate gives rise to patterns with larger amplitude. 


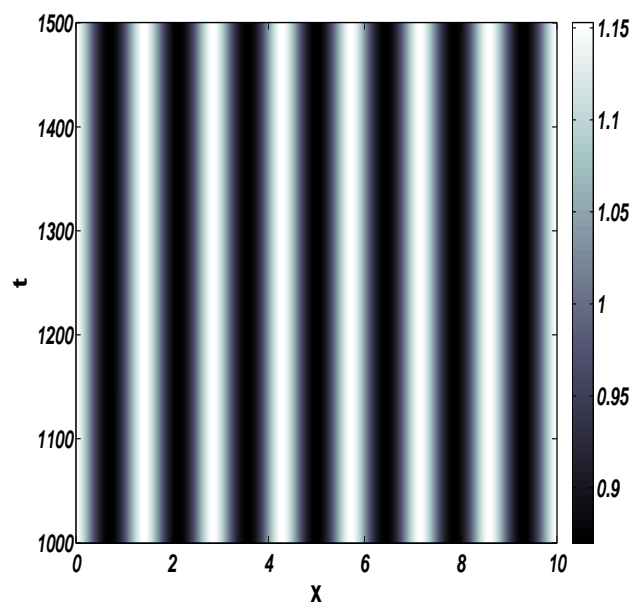

(a)

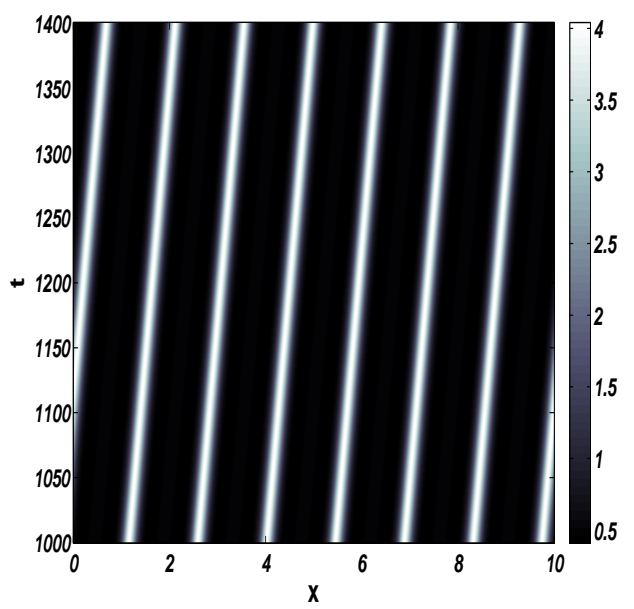

(b)

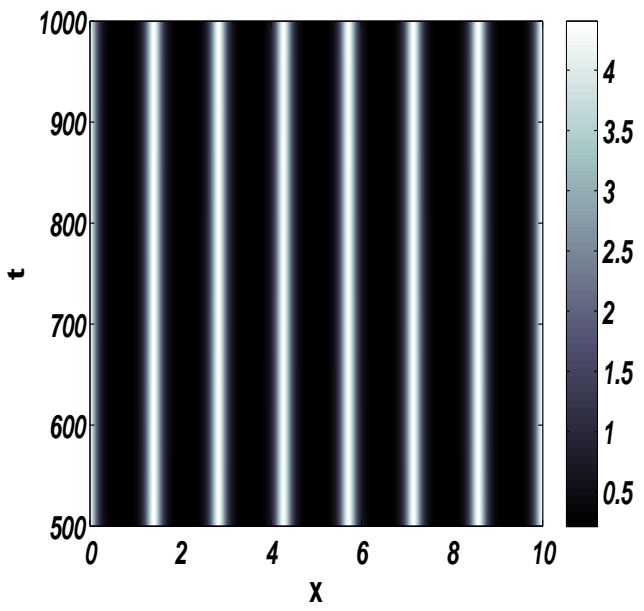

(c)

FiguRE 1. Stationary and traveling waves. Panel (a) illustrates stationary waves for the parameter set $\mathbf{P} 1$ with the initial condition $i(0.01)$ and the diffusion rate $\beta=0.1768$. Similarly, panel (b) shows traveling waves for the parameter set P2 with the initial condition $i(0.01)$ and the diffusion rate $\beta=0.0910$. Panel (c) is obtained from parameter set $\mathbf{P} 1$ with the diffusion rate $\beta=0.0910$

\subsubsection{Colony formation beyond the stability boundary}

Here, we numerically investigate the patterns away from the stability boundary using kernel function specified by $\mathbf{P 3}$.

Figure 2 (a) illustrates the solution $u$ for the parameter set P3 with the initial condition $i(0.001)$ and $\beta=0.001$. The amplitude levels of the solution is given in Figure 2 (b). As seen from Figure 2 (a), the computed patterns are not stationary or traveling wave type. We observe stationary wave islands for a while, and then the islands jump to left and forms a new colony. While parent islands vanish, displaced colonies form and they continue to propagate like stationary waves. 


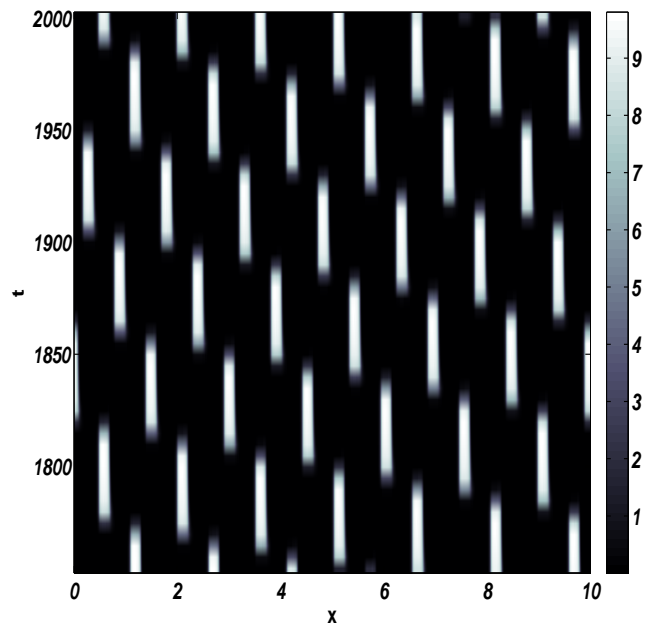

(a)

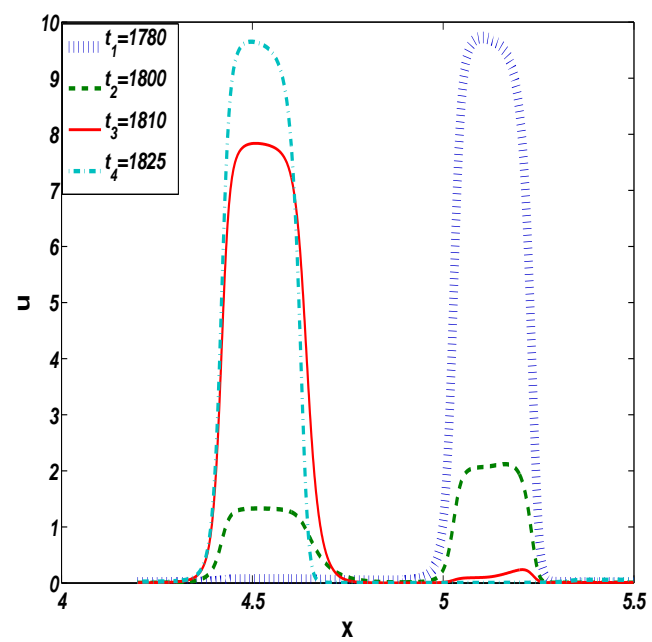

(b)

Figure 2. Regular colony formation. Panel (a) illustrates solution $u(x, t)$ for parameters P3, initial condition $i(0.001)$ and $\beta=0.001$. Panel (b) shows the jump of a stationary wave around $x=5.1$ with the parameters given in (a).

In order to further investigate the formation of colonies, we consider discrete time plots of $u$ with parameters given in Figure 2 (b). We plot $u$ for four nonuniformly spaced values of $t$. From time $t_{1}$ to $t_{2}$, there is a decrease in the amplitude of the solution around $x=5.1$, and there is a visible colony formation around $x=4.5$. As the parent island near $x=5.1$ decreases in amplitude, the new colony forms and grows in amplitude between times $t_{2}-t_{3}$. Finally a new colony with full amplitude forms while the parent island vanishes at time $t_{4}$.

We would like to note that colony formation is observed in $[14,17]$ for other models with nonlocal interaction terms. Solutions to these models, on the contrary, exhibit traveling wave type colony islands. Colony forming patterns presented in Figure (2) (a) and above mentioned studies share one common property: the change in amplitude occurs only when a new colony forms or vanishes. Thus, one can observe solutions exhibiting stationary or traveling wave islands and the amplitudes of these colony islands increase as they start forming and decrease as they vanish. Thus, we call the solutions shown in Figure 2 (a) as regular colony formation.

Nontheless, our computations show that one can observe more complicated changes in the amplitudes of colony islands. To this extent, consider the parameter set P3 with the same initial condition $i(0.001)$ and a different diffusion rate $\beta=0.0001$. Solution $u$ is presented in Figure 3 (a) showing that the amplitudes of colony islands change in time. This fact becomes more clear in Figure 3 (b) which shows that the change in amplitude within each colony is sinusoidal. Thus, one can observe more complicated amplitude changes than just increases and decreases at the starting and ending regions of a colony island.

Another interesting observation is that the direction of jumps in Figure 2 (a) and the direction of jumps shown in Figure 3 (a) changes too. Since the only differece between these figures is the nonlocal diffusion rate, it is clear that the direction of jumps depends on neither dispersal nor interaction kernels alone but it also depends heavily on the diffusion rate $\beta$.

In the two dimensional system, studied in [17], formation of colonies can be explained by the asymmetry of interaction kernel and interspecific competition. Here, the latter reason can be replaced by nonlocal dispersal. Observe that in our simulations, we chose our dispersal and interaction kernels such that their extent of asymmetries $a_{1}$ and $a_{2}$ have different signs i.e. dispersal kernel favors positive direction 


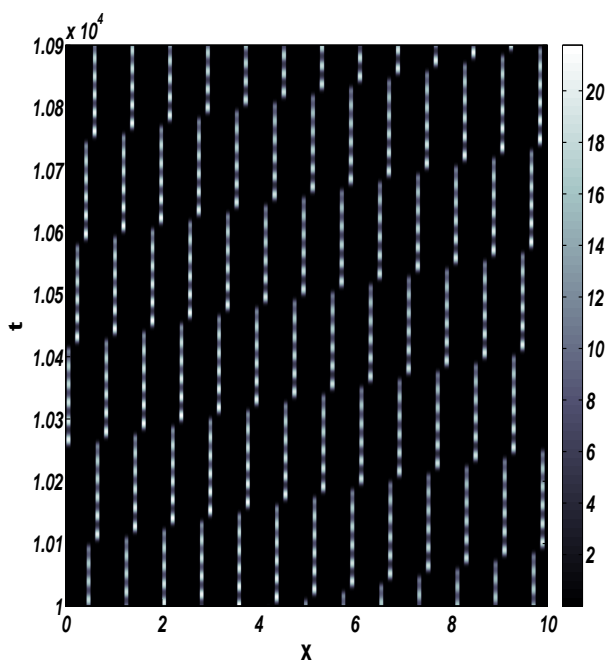

(a)

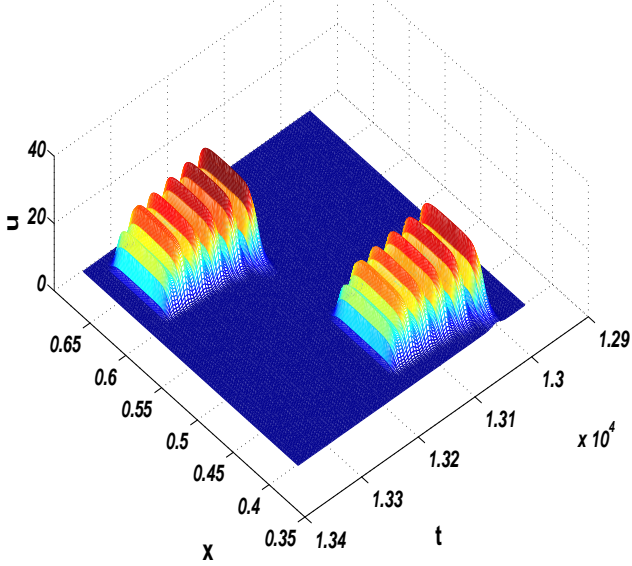

(b)

Figure 3. Colony formation with oscilating amplitude. Panel (a) shows solution $u(x, t)$ for parameters P3, initial condition $i(0.001)$ and $\beta=0.0001$. Panel (b) shows a specific jump of a colony island with the same parameter set.

while interaction kernel favors negative direction in space. Our simulations suggest that there is no colony formation if both kernels favor the same direction, but we see formation of periodic traveling waves. These simulation results indicate that one can observe colony formation as long as interaction and dispersal kernels favor different directions in space.

\section{Weakly Nonlinear Analysis}

The linear stability analysis is only valid for small time and infinitesimal perturbations. For large time nonlinear terms affect the growth of unstable modes. In this section, we consider model (1.1) and obtain Stuart-Landau equation that gives information about the qualitative behavior of the solutions.

\subsection{Analytical results}

We are interested in the stability of the homogenous solution $u=1$ near the critical nonlocal diffusion rate $\beta_{0}$ with wrap around boundary conditions on $I=[0, L]$. Our aim is to find a weakly nonlinear solution to (1.1) by multiple-scales perturbation expansion. Consider a slow time scale $\tau=\varepsilon^{2} t$ and expand $u$ as well as the bifurcation parameter $\beta$ as

$$
u=1+\varepsilon u_{1}(x, t, \tau)+\varepsilon^{2} u_{2}(x, t, \tau)+O\left(\varepsilon^{3}\right)
$$

and

$$
\beta=\beta_{0}+\varepsilon^{2} \mu
$$

for $0<\varepsilon \ll 1$ and $\mu= \pm 1$. Here $\mu$ determines the direction of the deviation from the critical nonlocal diffusion rate $\beta_{0}$. A direct implication of the linear stability analysis is that $u=1$ is unstable if $\mu=-1$. However, we will show that instabilities may occur even for $\mu=1$ for some kernel functions.

Define the spatially periodic family of linear waves as follows:

$$
E_{m}=\exp \left(i m\left(k_{c} x+\omega_{0} t\right)\right)
$$


for $\omega_{0}=\mathcal{I}\left[\lambda\left(\beta_{0}, k_{c}\right)\right]$ and $m \in \mathbb{Z}$.

Since our model contains a quadratic term, $E_{1}$ and $E_{-1}$ terms only couple with $E_{0}$ and $E_{ \pm 2}$. Thus we consider the ansatz (4.1) with the following functions:

$$
u_{1}(x, t, \tau)=A(\tau) E_{1}+\text { c.c. }
$$

and

$$
u_{2}(x, t, \tau)=A_{0}(\tau) E_{0}+A_{2}(\tau) E_{2}+c . c .
$$

where $A, A_{0}$ and $A_{2}$ are functions of $\tau$ only and c.c. stands for complex conjugate.

Using the ansatz (4.1) in (1.1), we obtain the following Stuart-Landau type equation:

$$
A_{\tau}=\Phi A+\Psi A|A|^{2} .
$$

Details of the derivation of (4.6) and the coefficients $\Phi$ and $\Psi$ are given in Appendix A.

Note that solutions to amplitude equations (4.6) are complex. We redefine the amplitude function as $A(\tau)=L(\tau) e^{i \theta(\tau)}$ with real terms $L(\tau)=|A(\tau)|$ and $\theta(\tau)$. Therefore, equation (4.6) can be written as following:

$$
\begin{aligned}
& \frac{d L}{d \tau}=\mu \mathcal{R}[\Phi] L+\mathcal{R}[\Psi] L^{3}, \\
& \frac{d \theta}{d \tau}=\mu \mathcal{I}[\Phi]+\mathcal{I}[\Psi] L^{2} .
\end{aligned}
$$

Steady state solutions of the equation (4.7) are given by $L_{0}=0$ and $L_{1}=\sqrt{-\mu \mathcal{R}[\Psi] / \mathcal{R}[\Phi]}$. The linear stability analysis of (4.7) suggests that the solution 0 is stable whenever $\mu \mathcal{R}[\Phi]<0$. Thus, it is clear from (A.6) that the steady state solution $u=1$ ( or $L_{0}$ ) is unstable for $\mu=-1$ and it is stable for $\mu=1$. This result is in accordance with the results of the linear analysis given in Section 3.1

Moreover, we need $L_{1}$ to be real i.e. $\mu \mathcal{R}[\Psi] \geq 0$. Thus the sign of $\mathcal{R}[\Psi]$ determines the direction of deviation from bifurcation parameter. One can categorize the possible bifurcations arising from (4.7) as follows:

1. If $\mathcal{R}[\Psi]>0$, then $\mu=-1$ and (4.7) describes a supercritical bifurcation with stable branch $\varepsilon L_{1}$ to the left of $\beta_{0}$.

2. If $\mathcal{R}[\Psi]<0$, then $\mu=1$ and (4.7) describes a subcritical bifurcation with unstable branch $\varepsilon L_{1}$ to the right of $\beta_{0}$.

In terms of (1.1), the first case implies that a supercritical pitchfork bifurcation arises from the constant density state $u=1$ to a spatially periodic pattern with steady state amplitude determined by $L_{1}$. In the second case, transitions to instabilities are discontinuous and $L_{1}$ determines the maximum amplitude of the initial conditions above which $u=1$ is unstable. In the next section, we give bifurcation diagrams, and numerically verify these results for the parameter sets P1-2.

\subsection{Numerical verification of analytical results}

In this section, we consider (1.1) with parameters $\mathbf{P} \mathbf{1}$ and $\mathbf{P 2}$. One can calculate the values of $\Psi$ for parameter sets P1-2 as $\Psi^{s w}=-0.2001$ and $\Psi^{t w}=0.2192+i 0.0677$ by using formula (A.7). The analytical results obtained in Section 4.1 suggest that transition to instability for stationary waves is supercritical while it is subcritical for traveling waves.

To be able to verify these results numerically, define the half amplitude of solutions to equation (1.1) by

$$
\alpha:=\frac{\max _{x \in I}\left\{u\left(x, t^{*}\right)\right\}-\min _{x \in I}\left\{u\left(x, t^{*}\right)\right\}}{2}
$$


for sufficiently large $t^{*}$. We study quantity $\alpha$ defined above and compare it to the numerical observations. Substitute the perturbation extension (4.1) with first two terms i.e. $u=1+\varepsilon u_{1}$ to (4.8). One can easily obtain

$$
\alpha=2 \varepsilon \mathcal{R}[A]
$$

We verify our analytical results obtained in Section 4.1 by numerical simulations. Consider the parameter sets P1-2 again. Figure 4 (a) shows the stable branch (black curve) obtained by using the analytical results and stable pattern amplitudes (black dots) for small perturbations of critical nonlocal diffusion rate $\beta_{0}$. As seen from the figure, nonlinear prediction (4.9) and the amplitude $\alpha$ obtained from numerical simulations of (1.1) stays pretty close for small values of $\varepsilon$.

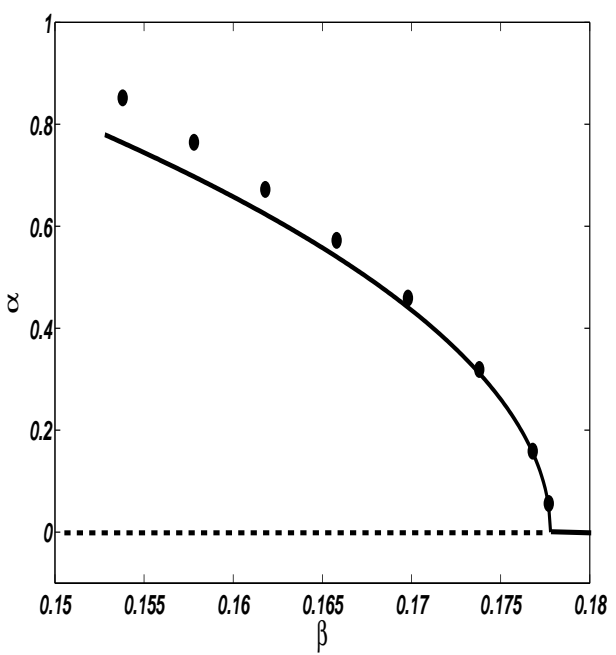

(a)

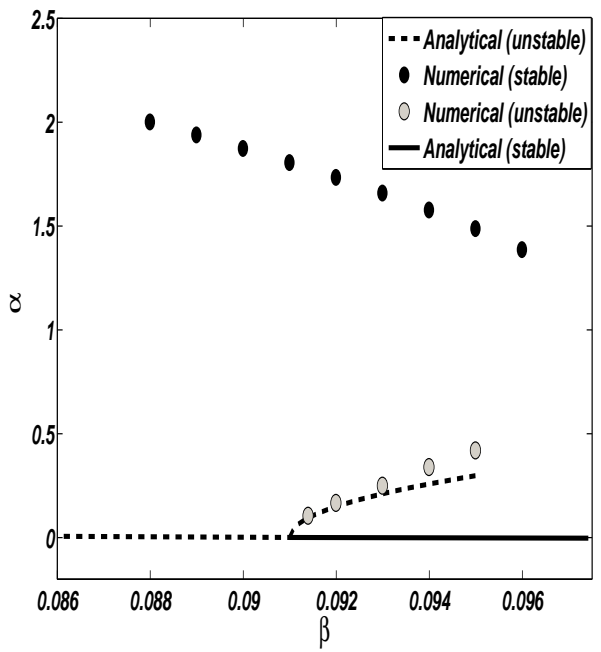

(b)

Figure 4. Numerical verification of analytical results obtained in Section 4.1 Panel (a) shows amplitude levels of stationary waves with parameters $\mathbf{P} \mathbf{1}$ around $\beta_{0}=0.1778$. Panel (b) illustrates amplitude levels of traveling waves with parameters P2 around $\beta_{0}=0.0910$.

On the other hand, Figure 4 (b) shows the validity of analytical results for subcritical bifurcations. The unstable branch (dashed curve) shown in Figure 4 (b) determines the maximum amplitude of initial conditions below which space homogenous equilibrium $u=1$ is stable. If the amplitude of the initial condition passes this maximum value then one can observe pattern formation whose amplitude levels are obtained numerically (gray dots).

More generally, one can easily identify the regions in which supercritical and subcritical bifurcations arise by employing the formula for $\Psi$ given in (A.7) for any kernel family. We consider a set of parameters given by $K_{1}=\mathcal{K}_{(1,0)}$ and $K_{2}=\mathcal{K}_{(d, a)}$ for $0<d \leq 4$ and $0 \leq a \leq 4$. Figure 5 identifies two different regions for this family. The region colored in black is the parameter set for which the subcritical bifurcations arise; and the white region represents the parameter set for the supercritical bifurcations. Basically, Figure 5 is the map representing $\operatorname{sign}(\Psi)$ where white and black regions represent positive and negative values of $\Psi$ given by (A.7), respectively. 


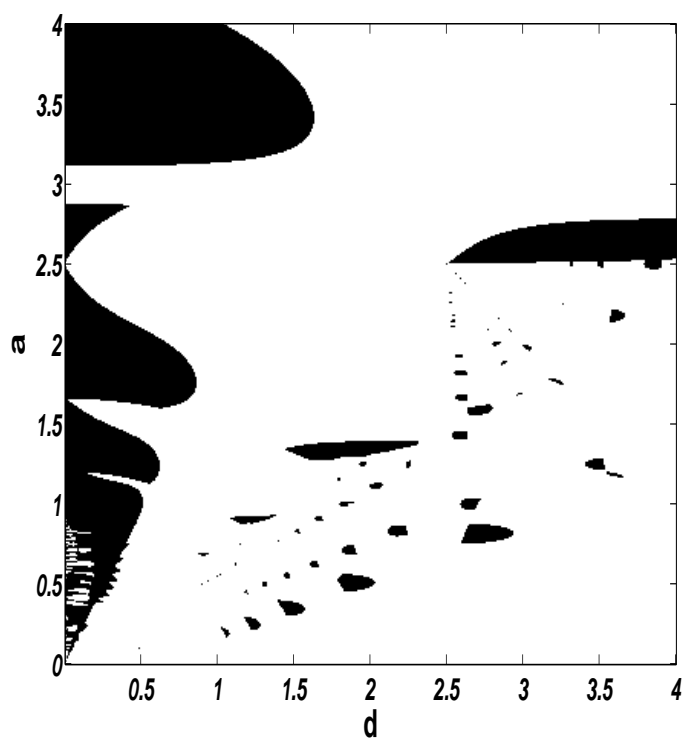

FiguRE 5. Bifurcation diagram for $K_{1}=\mathcal{K}_{(1,0)}$ and $K_{2}=\mathcal{K}_{(d, a)}$. White region represents parameters where transition from diordered to ordered behavior is supercritical while black region represents parameters for which the transitions are subcritical.

\section{Conclusions}

In this study, we considered a simple intraspecific competition model with nonlocal diffusion and interaction. We assumed that both individuals' movement and interactions are governed by probability kernels modeling distance-dependent long range interactions and movement patterns. These nonlocal terms were assumed to depend on two parameters that are the range of nonlocality and the extent of asymmetry.

We analyzed the deterministic model near and away from stability boundaries. Our main interest was to study emergence of new patterns and transitions to instability. Related to these objectives, our main results can be summarized as follows.

1. We investigated the necessary conditions to destabilize the space homogenous solution $u=1$ in terms of the Fourier transforms of interaction and dispersal kernels and the diffusion rate $\beta$. We observed that solution $u=1$ becomes unstable whenever Fourier transform of the interaction kernel takes negative values and the diffusion rate is sufficiently small.

2. Our simulations using the uniform kernel family (3.3) indicate that one can only observe traveling and stationary waves near the stability boundary. Similar patterns were observed for nonlocal Fisher equation [7]. We also observed a discrepancy between amplitudes of the patterns near the stability boundary. In particular, our numerical examples indicate the emergence of small and large amplitude patterns for some kernel functions near the stability boundary.

3. To be able to explain the discripancy between the amplitudes, we further investigated the model via Stuart-Landau theory. Using the multi-scales analysis, we show that transition to instability can be subcritical or supercritical depending on the nonlocal interaction and dispersal kernels. We also numerically verify the results of nonlinear prediction and give a bifurcation diagram for a family of interaction kernels.

4. In our computational studies, we observed the emergence of different types of colony formations whenever directions favored by the interaction and dispersal kernels are different. We find solutions exhibiting regular colony formation i.e. a colony is formed just ahead of a stationary wave island where it 
grows as a parent island and decays. We also observe oscillating colony formation for which amplitude change within any colony island is sinusoidal. In addition, we observed that the direction of jumps can be different with the same kernel functions depending on the value of the diffusion rate $\beta$.

Finally there are a few interesting issues that should be further explored and extended. In this paper, we focused on equation (1.1) in one dimensional habitat. We believe that computational analysis of this equation away from the stability boundary is worth investigating in two spatial dimensions. Similarly, weakly nonlinear analysis can be applied to two space dimensions following [18] to extend our analysis or to conclude the existence (if exists at all) of qualitatively different patterns. In addition, the same model with time dependent nonlocalities or time delays may give rise to emergence of more complex patterns. Such extensions may be useful in many cases. For instance, one can study seasonal effects [10], or the effect of regeneration using time periodic or delayed nonlocality on the emergence of patterns. Lastly, one can study the effect of nonlocal diffusion in two dimensional systems considered in $[1,15,17]$.

Appendix

\section{A. Derivation of Stuart-Landau equation}

Consider the linear operator associated to equation (1.1):

$$
L[u]=u_{t}-\beta_{0}\left(K_{1} * u-u\right)+K_{2} * u .
$$

We apply the linear waves $E_{n}$ given in (4.3) to this operator. This yields:

$$
\mathcal{L}_{n}=i n \omega_{0}-\beta_{0}\left(\hat{K}_{1}\left(n k_{c}\right)-1\right)+\hat{K}_{2}\left(n k_{c}\right) .
$$

Following [3], one can use $\mathcal{L}_{1}$ and Fredholm alternative theorem throughout the analysis. However, imposing the boundary conditions will be enough as done in [19] since we have a single equation rather than a system of equations.

Here we would like to note that the identity $K * E_{n}=\hat{K}\left(n k_{c}\right) E_{n}$ is used implicitly for $n \in \mathbb{Z}$.

By plugging the ansatz (4.1), one gets at the level $O(\varepsilon)$

$$
A(\tau) \mathcal{L}_{1} E_{1}+A^{*}(\tau) \mathcal{L}_{-1} E_{-1}=0
$$

where $A^{*}$ denotes the complex conjugate of $A$. Requiring the coefficients of the terms $E_{ \pm 1}$ are 0 at this level, one obtains the linear problem (3.2).

At $O\left(\varepsilon^{2}\right)$ level, we get

$$
\begin{aligned}
\left(A^{2} \hat{K}_{2}\left(k_{c}\right)+\mathcal{L}_{2} A_{2}\right) E_{2} & +\left(A_{0}+|A|^{2}\left(\hat{K}_{2}\left(k_{c}\right)+\hat{K}_{2}\left(-k_{c}\right)\right)\right) \\
+ & \left(A^{* 2} \hat{K}_{2}\left(-k_{c}\right)+\mathcal{L}_{-2} A_{2}^{*}\right) E_{-2}=0
\end{aligned}
$$

where we assumed $A_{0}$ is real. One can easily see that this assumption does not reeally affect the result. By requiring the coefficients of the terms $E_{0}$ and $E_{2}$ are 0 , we obtain:

$$
\begin{aligned}
& A_{0}=-2 \mathcal{R}\left[\hat{K}_{1}\left(k_{c}\right)\right]|A|^{2} \\
& A_{2}=-\frac{\hat{K}_{2}\left(k_{c}\right)}{\mathcal{L}_{2}} A^{2} .
\end{aligned}
$$

At $O\left(\varepsilon^{3}\right)$, one gets

$$
P^{(1)} E_{1}+P^{(2)} E_{3}+c . c=0 .
$$

where

$$
P^{(1)}=A_{\tau}-\mu\left(\hat{K}_{1}\left(k_{c}\right)-1\right) A+\left(1+\hat{K}_{2}\left(k_{c}\right)\right) A A_{0}+\left(\hat{K}_{2}\left(-k_{c}\right)-\hat{K}_{2}\left(2 k_{c}\right)\right) A^{*} A_{2}
$$


and $P^{(2)}$ is a function of Fourier transforms of the kernels and $A_{2}$. By imposing the boundary conditions again, we need $P^{(1)}$ to be 0 . Using this equality together with the equations (A.3) and (A.4), we get the equation (4.6) with the parameters:

$$
\Phi=\left(\hat{K}_{1}\left(k_{c}\right)-1\right)
$$

and

$$
\Psi=2 \mathcal{R}\left[\hat{K}_{2}\left(k_{c}\right)\right]\left(1+\hat{K}_{2}\left(k_{c}\right)\right)+\frac{\hat{K}_{2}\left(k_{c}\right)\left(\hat{K}_{2}\left(2 k_{c}\right)+\hat{K}_{2}\left(-k_{c}\right)\right)}{\mathcal{L}_{2}}
$$

Acknowledgements. The author is greatful to an anonymous referee for a number of helpful suggestions for improvement in the article.

\section{References}

[1] N. Britton. Aggregation and the competitive exclusion principle. J. Theor. Biol. (1989), 136 (1), $57-66$.

[2] R. S. Cantrell, C. Cosner. Spatial ecology via reaction-diffusion equations. John Wiley \& Sons, 2004.

[3] R. Eftimie, G. de Vries, M. Lewis. Weakly nonlinear analysis of a hyperbolic model for animal group formation. J. Math. Biol. (2009), No.59 (1), 37-74.

[4] M. Fuentes, V. Kuperman, V. Kenkre. Nonlocal interaction effects on pattern formation in population dynamics. Phys. Rev. Lett. (2003), No. 91 (15), 158-104.

[5] M. Fuentes, V. Kuperman, V. Kenkre. Analytical considerations in the study of spatial patterns arising from nonlocal interaction effects. J. Phys. Chem. B (2004), No. 108 (29), 10505-10508.

[6] S. Genieys,N. Bessonov, V. Volpert. Mathematical model of evolutionary branching. Math.Comput. Model. (2009), No. 49 (11), 2109-2115.

[7] S. Genieys, V. Volpert, P. Auger. Pattern and waves for a model in population dynamics with nonlocal consumption of resources. Math. Model. Nat. Phen. (2006), No. 1 (01), 63-80.

[8] S. A. Gourley. Travelling front solutions of a nonlocal Fisher equation. J. Math. Biol. (2000), No. 41(3), $272-284$.

[9] E. E. Holmes, M. Lewis, J. Banks, R. Veit. Partial differential equations in ecology: spatial interactions and population dynamics. Ecology (1994), 17-29.

[10] J. Yu, M. Lewis. Seasonal influences on population spread and persistence in streams: spreading speeds. Journal of mathematical biology (2012), No. 65.3, 403-439.

[11] V. Hutson, S. Martinez, K. Mischaikow, G. Vickers. The evolution of dispersal. J. Math. Biol. (2003), 47 (6), $483-517$.

[12] F. Lutscher, E. Pachepsky, M. Lewis. The effect of dispersal patterns on stream populations. SIAM J. Appl. Math. (2005), No. 65 (4), 1305-1327.

[13] J. D. Murray. Mathematical biology II: spatial models and biomedical applications. Springer, 2003.

[14] B. Perthame, S. Genieys. Concentration in the nonlocal fisher equation: the hamilton-jacobi limit. Math. Model. of Nat. Phen. (2007), No. 2 (04), 135-151.

[15] B. Segal, V. A. Volpert, A. Bayliss. Pattern formation in a model of competing populations with nonlocal interactions. Physica D (2013), No. 253, 12-22.

[16] J. Stuart. On the non-linear mechanism of wave disturbances in stable and unstable parallel flows. part $i$. J. Fluid Mech. (1960), No. 9, 152-171.

[17] M. Tanzy, V. A. Volpert, A. Bayliss, M. Nehrkorn. Stability and pattern formation for competing populations with asymmetric nonlocal coupling. Math. Biosci. (2013), No. 246 (1), 14-26.

[18] A. E. Tikhomirova, V. A. Volpert. Nonlinear dynamics of endothelial cells. Appl. Math. Let. (2007), No. 20 (2), 163-169.

[19] C, M. Topaz, A. L. Bertozzi, M. Lewis. A nonlocal continuum model for biological aggregation. B. Math. Biol. (2006), No. 68 (7), 1601-1623.

[20] H. Uecker. Amplitude equations an invitation to multi-scale analysis; Lecture given at the International Summer School Modern Computational Science 2010. 